\title{
Eye-position aftereffects of backward head tilt manifested by illusory visual direction
}

\author{
WAYNE L. SHEBILSKE and LINDA A. FOGELGREN \\ University of Virginia, Charlottesville, Virginia 22901
}

\begin{abstract}
Three experiments showed posttest-minus-pretest shifts in subjective straight-ahead eye position when subjects read for 3,6 , or $9 \mathrm{~min}$ with their heads tilted back $20^{\circ}$ from upright. These shifts were significant relative to control conditions in which subjects read with their heads upright. All subjects read with the same straight-ahead eye-in-head position. Variability-reducing procedures were developed to provide better measures over Experiments 1, 2. and 3. Explanations in terms of deliberate compensation, head-position asymmetries, eye-position asymmetries, and progressive error were ruled out. It was hypothesized that the shifts were caused by negative aftereffects of compensation for the doll reflex. The doll reflex rotates the eyes down without central registration. causing an upward illusory shift of visual direction similar to what is caused by wedge prisms. Perceptual-motor adaptation to this shift, i.e., doll adaptation, causes an illusory shift in the opposite direction when the head is returned to upright.
\end{abstract}

When a person is tilted back in the median plane, the doll reflex causes a sustained elevation illusion analogous to that produced by base-down wedge prisms (cf. Ebenholtz \& Shebilske, 1975). If the appropriate informational discordance is present when a prism is worn, sensorimotor compensation reduces the displacement illusion and creates a negative aftereffect. Will comparable negative aftereffects occur if informational discordance is present during prolonged head tilts? This question is explored in the following experiments.

Doll eye movements are compensatory vertical rotations that occur when the head is tilted in the median plane; the eyes rotate downward with backward head tilts and upward with forward tilts. They were observed in adults under controlled conditions in 1934 by Schubert and Brecher and again in 1953 by DeWit. However, parametric measures were not reported until 1975 by Ebenholtz and Shebilske, who showed doll eye movements to be proportional to vestibular output, suggesting that reflexive otolithic innervations cause the counterrolling.

The procedure used by Ebenholtz and Shebilske controlled differences in voluntary eye movements and felt body position. Voluntary eye movements were equated at each body-head tilt by asking subjects to choose a direction of gaze perpendicular to their frontal plane, i.e., straight ahead. They were asked to choose from a vertical array of lights that one which was in their line of sight when their eyes

This paper was supported by NIH Subgrant BSS-5 505RR 07094-09. We thank Mark King, Arthur Kreiger, and Clyde Smoot for help in apparatus construction, data collection, and tabulation. Requests for reprints should be sent to Wayne L. Shebilske, Department of Psychology, Gilmer Hall, University of Virginia, Charlottesville, Virginia 22901 were in the normal straight-ahead position in their heads. These measures were independent of felt body position since the same eye in head position was requested regardless of body position.

These measures also bear upon the visual consequences of doll eye movements. The fact that lower targets were chosen when the head was tilted back implies that an actually straight-ahead light must have looked higher. This inferred elevation illusion is consistent with a similar illusion found in judgments of the horizon (e.g., Cohen, 1973; Schone, 1964), and is also supported by direct reports of subjects (cf. Ebenholtz \& Shebilske, 1973) and by unpublished pointing measures in our lab. The fact that apparent elevations are not noticed when heads are tilted in everyday activities is reminiscent of illusory slants which are observed in darkness but not in structured settings (cf. Wade, 1970). We assume that more than one informational system contributes to the perception of direction and slants and that higher order structural systems dominate. By this account, the lower order systems register their erroneous values during head tilts whether in light or dark, but their effects are seen only in the absence of structural information.

Doll eye movements and the associated elevation illusion must be sustained for at least a couple of minutes since the measures of Ebenholtz and Shebilske were taken after a 1-min rest period in each body-head position and the measures took about another minute. In this respect, the doll reflex may be like another otolithic eye-movement reflex, countertorsion, which does not diminish over intervals as long as $\mathbf{4 5} \mathrm{min}$ (cf. Howard \& Templeton, 1966).

The question of whether sustained head tilts cause negative aftereffects was probed in the present study 
in which we attempted to introduce sensorimotor and intramodal discordance with the head tilted. Subjects performed eye movements against a structured background with their heads tilted back in an experimental condition and with their heads upright in a control condition. Eye movements were included in an attempt to introduce sensorimotor discordance when the head was tilted. The intended eye movements were always along a horizontal path so that the expected reafference should have been horizontal retinal displacement. The relationship between the expected and actual sensory consequences of the self-produced eye movements may have been disrupted by the doll reflex. Eye position must be registered correctly for intended saccades to land on target because the effect of a particular muscle contraction varies depending on starting eye position (Krewson, 1950). Consequently, the doll reflex will cause saccades to land off target if the reflexive innervations are not registered appropriately. This, in turn, would produce sensorimotor discordance between the expected and actual reafference. The effects of the doll reflex on saccadic dysmetria remain to be tested. However, if sensorimotor discordance was present, it should have promoted adaptation according to the results of McLaughlin (1967) and Pola (in press), who found that discordant retinal feedback causes parametric adjustment. In addition, a structured background was included in an attempt to introduce intramodal discordance when the head was tilted. According to the hypothesis given above, lower order and higher order systems for visual direction are in conflict during head tilts because of the aberrant effects of the doll reflex on the lower order system. Both of these postulated sources of discordance stem from the doll reflex and therefore should have been absent in the control condition where heads were upright.

The specific test and exposure procedures were determined not only by an attempt to provide informational discordance, but also by the need to control four potential artifacts. (1) Deliberate compensation was controlled by using negative aftereffects rather than adaptation as the response measure. Identical tests were taken with the head upright before and after exposure periods. Four tests were tried in the first experiment and the one used by Ebenholtz and Shebilske (1975) proved to be the best. On this test, subjects look lower when their heads are tilted back so that the predicted negative aftereffect was for subjects to look higher on posttests than on pretests. Subjects might simulate adaptation by consciously correcting, but it is unlikely that deliberate compensation could explain negative aftereffects. (2) Maintained head rotations produce aftereffects when subjects point at a straightahead target (Ebenholtz, 1976). However, this should not have influenced the negative aftereffect assuming that subjects followed the instructions to choose the same eye-in-head position regardless of head position. (3) Slight asymmetries in direction of gaze cause posttest-minus-pretest shifts in apparent visual direction (e.g., Ebenholtz, 1976; Ebenholtz \& Wolfson, 1975; Paap, 1975). There is little value in postulating special adaptation effects when simple fixation asymmetries can explain the results (cf. Ebenholtz, 1974). Therefore, the present study followed the rationale of some prism experiments that have controlled asymmetry effects by equating eye-in-head position for the control and experimental conditions (e.g., Freedman, 1968; Howard, 1968). (4) A last function of the control condition was to take into account progressive errors from factors like warmup, practice, and/or fatigue. Since these could increase or decrease negative aftereffects, the absolute value of shifts in the experimental condition is a less appropriate measure than the shift in the experimental condition relative to the shift in the control condition.

\section{GENERAL METHOD}

\section{Subjects}

In all three experiments, the subjects were undergraduate students at the University of Virginia who were either paid volunteers or who participated in partial fulfillment of course requirements. Experiments 1, 2, and 3 had 24, 32, and 16 students, respectively, none of whom knew the hypothesis being tested. They were assigned randomly to conditions upon their arrival at the laboratory.

\section{Apparatus}

Two visual display units were used. Each one had a chinrest and forehead support that could be adjusted to put the head at the appropriate distance, height, and tilt. The latter was measured by an inclinometer (Pro No. 30), which was fastened to the head parallel to a line running from the outer canthus to the center of the ear canal. This measure approximated Reid's basline, a standard anatomical reference. We adopted the convention of calling tilts with the canthus end up and down "positive" and "negative," respectively.

The test display consisted of a vertical semicircular array of illuminated dots which were $1.316 \mathrm{~cm}$ in diameter $\left(.67^{\circ}\right)$ and located $.476 \mathrm{~cm}$, or $1^{\circ}$, apart at the center. The dots were uniformly illuminated, and intensity was controlled by a rheostat. The array covered an arc of about $170^{\circ}$ so that it extended above and below the visual field. The testing display was placed at a distance of $26.98 \mathrm{~cm}$ from the observer's eyes which was equal to the radius of the perimeter. This distance required a convergence angle of approximately $14^{\circ}$ when any dot was fixated. A moveable mask, attached to the left side of the perimeter, followed a path directly over the dots, occluding one at a time. A small wheat bulb could illuminate a dot at the center of the mask. The mask dot was the same size and intensity as the perimeter dots and was controlled by a separate switch. A movable pointer was attached to the right side of the perimeter and a measurement scale in degrees was inscribed on the front.

The exposure display unit had two settings. Stimuli in the control conditions were presented in a vertical plane at a distance of $32.4 \mathrm{~cm}$ from the observer. This distance is neutral with respect to disjunctive asymmetry aftereffects which might develop at other distances (Ebenholtz \& Wolfson, 1975). Stimuli in the 
experimental conditions were presented at the same distance, but $20^{\circ}$ higher and in a plane tilted $20^{\circ}$ top toward the subject. In this way, both groups viewed stimuli with the same eye-in-head position.

\section{Procedure}

The three experiments had different procedural details which will be discussed in separate subsections before each experiment. There was always a pretest before the first exposure period and an identical posttest after each of three periods. Each exposure period lasted $3 \mathrm{~min}$, so that posttests were taken after 3,6 , and 9 min of total exposure.

Experiments 1, 2, and 3 used different combinations of four kinds of tests: multilight-visual, unilight-visual, multilightmanual, and unilight-manual. All wete obtained in an otherwise dark room. During multilight-visual tests, the perimeter dots were on and the mask dot was off. The experimenter adjusted the mask until the subject indicated that it covered the dot which looked straight ahead. The subjects always chose the apparent straight-ahead light before masking and were instructed not to change their minds once masking started. This test was identical to the one used by Ebenholtz and Shebilske (1975). During unilightvisual tests, the perimeter dots were off and the mask dot was on. The experimenter adjusted the mask dot until the subject indicated that it looked straight ahead. The subjects were instructed not to follow the mask dot all the way from its starting position. Rather, they were told to look straight ahead and not to look at the dot until it got close to their line of sight. Once they looked at it, they were free to tell the experimenter to move it up and down until their eyes felt like they were in their straight-ahead position. During the multilight-manual test, the perimeter lights were on and the mask dot was off. The subjects put their right thumbs on the pointer (about 1 in. from the dots) and pointed to the dot that appeared straight ahead. During the unilateralmanual test, the perimeter lights were off and the mask dot was on. The mask dot was set at the objective straight-ahead position, and the subjects attempted to point at it with their thumbs. This test was similar to the one used by Ebenholtz (1976). The response measure on all four tests was taken as the average of two successive settings with different up-down starting positions for the mask, mask dot, or pointer. Starting positions were determined randomly for all subjects.

\section{EXPERIMENT 1}

\section{Method}

Preliminary procedures. Preliminary procedures were required to attain the desired head tilt and eye-in-head position for both displays. For the test display, the head tilt was set at $+15^{\circ}$, the average of 10 pilot subjects who were asked to put their heads in a "natural" position. The eye-in-head position was controlled by changing the height of the head after the appropriate tilt was obtained. The height was set so that the center of the stimulus was on a horizontal line at eye level.

For the exposure display, head tilt depended on the condition. In control conditions, it was $+15^{\circ}$, and in experimental conditions, it was $+35^{\circ}$. In other words, head tilt was the same during test and exposure in control conditions and $20^{\circ}$ farther back in experimental conditions during exposure. The eye-in-head position was set by putting the center of the target on a horizontal line at eye level for control conditions and on a line tilted up $20^{\circ}$ from the horizontal at eye level in experimental conditions. Because the head was tilted back $20^{\circ}$ more in the experimental conditions, this procedure equated eye-in-head position by putting the eyes in the actual straight-ahead position for both groups.

Test periods. Four measures were obtained during each test period. Half of the trials obtained scores on multilight-visual and unilight-visual tests during each test period, while half obtained scores on multilight-manual and unilight-manual tests.
Exposure periods. During the three exposure periods, subjects read typed passages about transportation, plants, and world population in that order. Each one measured $9.5 \times 9.5 \mathrm{~cm}$ and was centered in the appropriate position for each condition. Subjects continued to read and reread aloud for $3 \mathrm{~min}$.

Filler task. Each session was divided into two parts separated by $5 \mathrm{~min}$. A filler task was used to minimize interactions between the two halves. In Experiment 1, the filler task was not strictly controlled. Subjects were asked to leave the laboratory and to return after $5 \mathrm{~min}$.

Design. Experimental and control conditions were betweensubjects factors. The factorial combination of two order factors, visual first or manual first and multilight first or unilight first, created four subgroups within each of the main groups. Exposure duration was a within-subjects variable. All subjects were tested after 3,6, and 9 min of total exposure time during each half of the experimental session. Four response measures were taken on each subject. One-half of the session measured visual tests; the other half measured manual. Each test period measured both multilight and unilight scores. Separate analyses were done for visual and manual measures. Multilight and unilight were treated as a second within-subjects variable for each analysis.

\section{Results and Discussion}

The manual measures yielded no significant main effects or interactions, which was probably due to adaptation of the outstretched arm causing increased variability. Therefore, manual results will not be presented.

The mean pretest scores were $-1.01^{\circ}$ and $-1.48^{\circ}$ for multilight-visual and unilight-visual. Pretest scores were subtracted from posttest scores to yield degrees of shifts which are plotted in Figure 1 after 3,6 , and $9 \mathrm{~min}$ of total exposure. A positive shift represents an upward change in subjective straightahead eye position, which is the predicted negative aftereffect of doll adaptation. The experimentalcontrol difference was significant, $F(1,22)=5.14$, $\mathrm{p}<.05$. The multilight-unilight factor had no main effect and did not enter into any significant interactions.

The results were encouraging; doll adaptation was supported by the predicted experimental-control difference on two separate measures. However, the downward shift of the control group suggested that progressive errors influenced the measures. Although these are unimportant assuming that they affected the experimental and control groups equally, cleaner measures seemed desirable for demonstrating a new adaptation effect. Therefore, Experiment 2 was planned.

\section{EXPERIMENT 2}

\section{Method}

Preliminary procedures. The main changes in preliminary procedures were the establishment of a subjective upright head position for each subject and the addition of warm-up trials. Each of these was designed to reduce progressive errors. Since doll eye movements go up or down depending on whether the head is tilted forward or backward, there must be some neutral head position. We attempted to estimate this position by asking sub- 


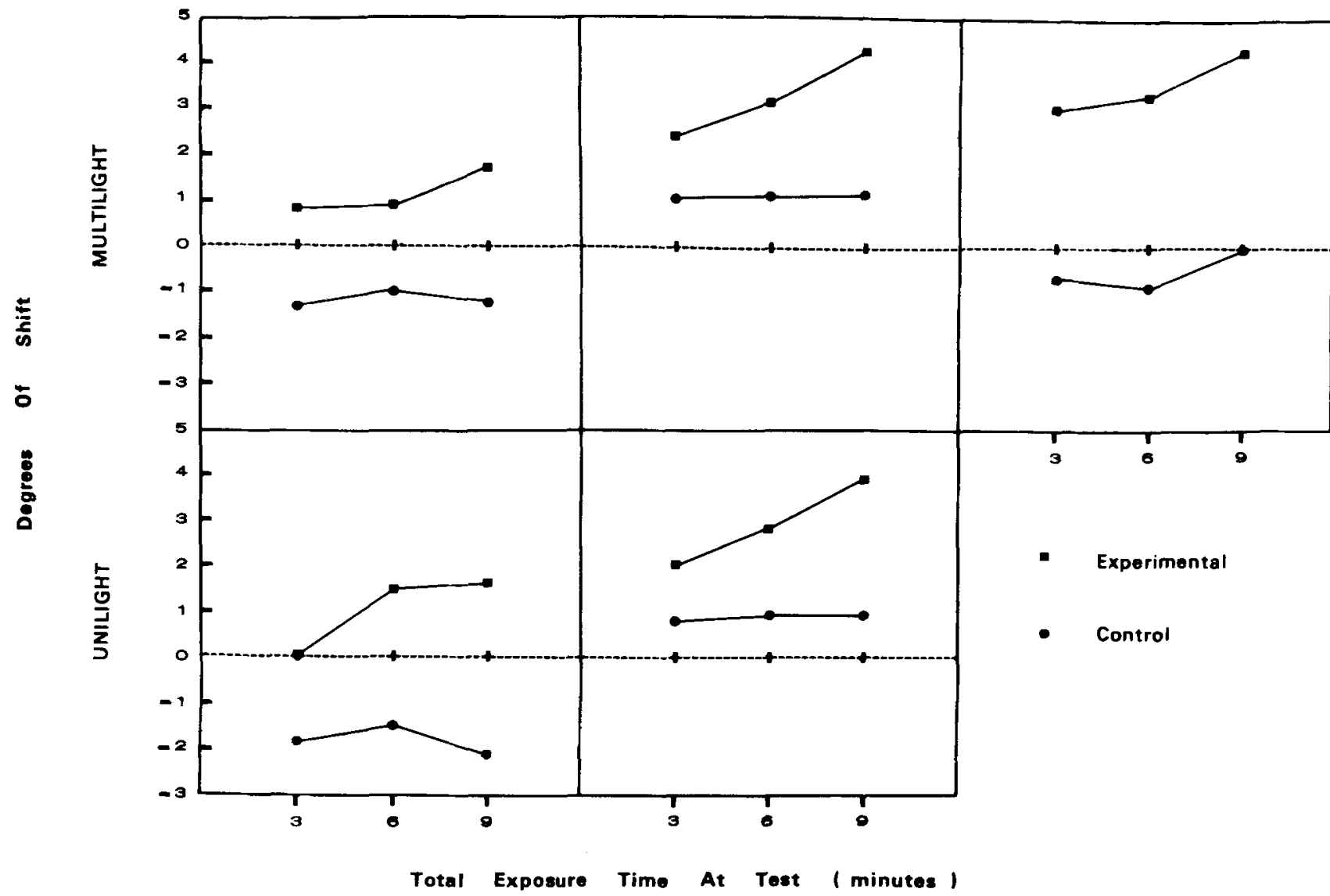

Figure 1. Degrees of posttest-minus-pretest shift for experimental and control conditions as a function of exposure time. Separate functions are shown for unilight and multilight response measures and for Experiments 1, 2, and 3. Positive and negative values indicate upward and downward shifts, respectively.

jects to put their heads in the "natural" upright position. They nodded their heads up and down three times between each estimate. The average of 10 trials was taken as their subjective upright head position. Their heads were held in this position for the test display and for the control exposure condition. The head position for the experimental exposure condition was $20^{\circ}$ back from that position. Eye-in-head position was controlled in the same way as in Experiment 1.

Eight warm-up trials (four multilight and four unilight) were given to measure progressive error and to bring it to an asymptotic level before the experiment.

Test periods. Manual measures were omitted because they were highly variable. In Experiment 2, multilight-visual and unilight-visual scores were obtained during each test period. Order of tests was balanced across subjects.

Exposure periods. Words on a memory drum replaced the written passage in order to control eye movements and direction of gaze. In Experiment 1, individual subjects finished the passage with their eyes in different places so that eye-in-head positions were variable. In Experiment 2, eye movements and direction of gaze were strictly controlled. Four-letter highfrequency words (chosen from the Thorndike-Lorge count) were typed on drums that displayed one word per second in a $1 \times 6.5 \mathrm{~cm}$ slit. Twenty-seven words appeared in a left, middle, and right repeating pattern, and were read aloud for $3 \mathrm{~min}$. Thus, all subjects made the same number of eye movements of the same size, and all ended with the same direction of gaze.
The slits was centered in the same position as the centers of the passages in Experiment 1. The vertical and $20^{\circ}$ planes in which the slits appeared were defined by checkerboard patterned panels.

Filler task. Each session was divided into two halves for experimental and control conditions separated by $5 \mathrm{~min}$, during which subjects were asked to leave the laboratory.

Design. The experimental-control factor was a within-subjects variable in Experiment 2. Experimental conditions were run in one half of each session and control conditions in the other half. Exposure duration $(3,6$, and $9 \mathrm{~min})$ was the only other withinsubjects factor. Multilight and unilight measures were analyzed separately. In each analysis, two order factors (multilight first or unilight first and experimental first or control first) were factorially combined as between-subjects variables.

\section{Results and Discussion}

It is difficult to assess the contribution of establishing a subjective upright head position for each subject. The mean head position that was felt to be upright was $+16.25^{\circ}$, averaged over all 32 subjects, with a standard deviation of $4.45^{\circ}$. As we expected, there was variability in what individuals considered to be a natural upright head position. It will take future experiments to determine if the subjective 
upright position is, in fact, neutral with respect to doll adaptation.

It is easier to see the value of the warm-up trials, which were analyzed in two blocks. For the multilight test, the mean subjective straight ahead was $-1.86^{\circ}$ and $-3.43^{\circ}$ for the first and second blocks; for the unilight test, the means were $-2.82^{\circ}$ and $-3.85^{\circ}$. These downward progressive errors were significant, $F(1,30)=12.89, p<.01$, and $F(1,30)=$ $7.24, p<.025$. The pretest scores for Experiment 2 were -3.31 and -3.62 for multi- and unilight tests, showing that most of the downward progressive error was eliminated by the warm-up trials. The experimental vs control conditions showed no main effects or interactions on screening or pretests, supporting the assumption that progressive errors affected the groups equally.

The negative aftereffects results for Experiment 2 are shown in the middle column of graphs in Figure 1. Again, the predicted negative aftereffect of doll adaptation was obtained for both response measures as indicated by the significant difference in shift for the experimental and control conditions $([F(1,28)=9.49, p<.01$, for multilight; $F(1,28)=$ $7.14, p<.025$, for unilight]. Additional support for doll adaptation was shown by a significant main effect of exposure duration $[F(2,26)=7.98, p<.01$, for multilight; $F(2,56)=5.70, p<.01$, for unilight] and a significant Exposure Duration by ExperimentalControl interaction $[F(2,56)=4.14, \mathrm{p}<.05$, for multilight; $F(2,56)=5.60, p<.01$, for unilight]. The amount of change in subjective straight-ahead eye position increased over time, with almost all of the increase being accounted for by the experimental condition.

The only other significant effect was an interaction between the experimental-control factor and the order of testing experimental and control conditions $[F(1,28)=8.13, p<.01$, for multilight; $\mathrm{F}(1,28)=13.91, \mathrm{p}<.01$, for unilight]. There was a much larger difference between the experimental and control conditions when the experimental condition was tested first. In fact, there was a negligible difference when the control condition was first. This problem, along with the upward shift in the control condition, motivated Experiment 3.

In principle, the upward shift for the control condition caused no problems of interpretation, since the critical comparison is the difference in shift between the control and experimental conditions. Still, we wanted to find a control condition that would cause no shift. Since left and right eye-position asymmetries cause left and right shifts, respectively, up and down asymmetries probably cause vertical shifts. And just as some head position must be neutral with respect to doll eye movements, some eye position must be neutral with respect to asymmetry effects. Perhaps the eyes shifted upward in Experiment 2 because the eye position was above the neutral point during exposure. The eye-in-head position was exactly straight ahead, but this may not be neutral. A better estimate might be the position that subjects feel to be straight ahead, which is about $-3.5^{\circ}$ according to the pretest of Experiment 2. If that is neutral, the actual straight-ahead eye position is too high, which would explain the upward shift.

\section{EXPERIMENT 3}

\section{Method}

Preliminary procedures. The two main changes in preliminary procedures were the establishment of a subjective straight-ahead eye-in-head position for the exposure displays for each subject and the use of better controls for preexperimental doll adaptation. The mean of each subject's warm-up settings was used for the subjective straight-ahead eye position. As an added step against asymmetry effects, subjects who had a mean subjective straight-ahead equal to or greater than $\pm 10^{\circ}$ were excluded. One subject was replaced because of this criterion. In order to reduce the effects of doll adaptation that might have developed during the apparatus adjustments, all adjustments that required backward head tilts were done first. Thus, any adaptation that developed would have had time to decay during the other apparatus adjustments and during the time the instructions were read.

Other aspects of the preliminary procedures were the same as Experiment 2 . The eye-in-head position was equated for experimental and control conditions, head tilt during test and control exposure was the "natural" position for each subject, and the experimental head tilt was $+20^{\circ}$ back from that.

Test periods. Experiments 1 and 2 used multiple response measures to determine which was the best way to measure doll adaptation. This information was gained at the expense of underestimating doll adaptation, since decay of adaptation probably occurred during the time required to take several measures. Therefore, Experiment 3, eliminated unilight measures. (The decision to eliminate unilight was based on its greater variability.) Hence, only multilight measures were taken during each test period, cutting test time in half.

Exposure periods. The exposure periods were identical to Experiment 2, except for the different eye-in-head position.

Filler task. Each session was divided into halves for experimental and control conditions, separated by $5 \mathrm{~min}$. In an attempt to eliminate the carryover effects observed in Experiment 2, the experimenter took subjects on a 5-min walk through the corridors on the same floor as the laboratory.

Design. Experimental and control conditions and exposure duration $(3,6$, and $9 \mathrm{~min})$ were factorially combined withinsubjects factors. Order of testing experimental and control was a between-subjects factor.

\section{Results and Discussion}

The mean "natural" head position was $15.24^{\circ}$, with a standard deviation of $4.45^{\circ}$. The controls for progressive error caused by preexperimental doll adaptation were effective since there was no progressive error over three blocks in the warm-up trials, $-3.59^{\circ},-3.44^{\circ}$, and $-3.41^{\circ}, \mathrm{F}<1$. There were no experimental and control interactions on screening and pretests.

The negative aftereffect results are shown in the right-hand graph in Figure 1. As expected, the upward control shift observed in Experiment 2 was 
eliminated by using the subjective straight-ahead eye-in-head position. In addition, measured amount of negative aftereffect was increased by using a shorter test.

The experimental-control difference was highly significant, $F(1,14)=33.35, p<.001$. The main effect of exposure duration was also significant, $F(2,28)=5.65, p<.01$. But the Exposure Duration by Experimental-Control interaction was not significant, $F<1$. Finally, in contrast to Experiment 2, there was not a significant interaction between the Experimental-Control factor and the Order factor, $\mathrm{F}(1,14)=2.11, \mathrm{p}>$.10. In Experiment 2, there was a negligible experimental-control difference when the control condition was tested first. In contrast, there was a $3.10^{\circ}$ difference in the predicted direction when the control condition was tested first in Experiment 3.

This is by far our cleanest measure of doll adaptation to date. The highly significant experimentalcontrol difference in Experiment 3 shows the success of the variability reducing steps developed over the three experiments; the use of individual subjective upright head position and individual subjective straight-ahead eye-in-head positions were probably the most important of these. Taken as a whole, the three experiments suggest that doll adaptation is a reliable, robust phenomenon.

\section{GENERAL DISCUSSION}

The answer to our original question is yes; when informational discordance is present during prolonged head tilts, negative aftereffects develop in judgments of visual direction. However, the fact that the present experiments were modeled after prism adaptation studies does not necessarily imply that doll adaptation has the same underlying mechanisms. Informational discordance is important in prism adaptation (e.g., Held, 1961; Wallach, 1968), so we included it in order to optimize the chances of obtaining a new kind of adaptation. However, the necessary and sufficient conditions for doll adaptation may be very different from those of other compensations. Optical devices, tendon crossings, and nerve transpositions introduce uncommon central-peripheral relationships, whereas head tilts produce an unregistered reflex that is continually present in everyday life. It remains for further research to identify the underlying mechanisms of doll adaptation.

\section{REFERENCES}

COHEN, M. M. Elevator illusion: Influences of otolithic organ activity and neck proprioception. Perception \& Psychophysics, 1973, 14, 401-406.

DEWIT, G. Seasickness (motion sickness): A labyrinthological study. Acta Otolorangology Supplement, 1953, 108, 7-56.

EBenholtz, S. M. The possible role of eye-muscle potentiation in several forms of prism adaptation. Perception, 1974, 3, 477-485.

Ebenholtz, S. M. Additivity of aftereffects of maintained head and eye rotations: An alternative to recalibration. Perception \& Psychophysics, 1976, 19, 113-116.

Ebenholtz, S. M. \& \& Shebilske, W. L. Instructions and the $\boldsymbol{A}$ and $E$ effects in judgments of the vertical. American Journal of Psychology, 1973, 86, 601-612.

Ebenholtz, S. M., \& Shebilske, W. L. The doll reflex: Ocular counterrolling with head-body tilt in the median plane. Vision Research, 1975, 15, 713-717.

Egenholtz, S. M., \& Wolfson, D. M. Perceptual aftereffects of sustained convergence. Perception \& Psychophysics, 1975, 17, 485-491.

Freedman, S. J. Perceptual compensation and learning. In S. J. Freedman (Ed.), The neuropsychology of spatially oriented behavior. Homewood, Ill: Dorsey Press, 1968.

HELD, R. Exposure-history as a factor in maintaining stability of perception and coordination. Journal of Nervous and Mental Disorders, 1961, 132, 26-32.

HowaRd, I. P. Displacing the optical array. In S. J. Freedman (Ed.), The neuropsychology of spatially oriented behavior. Homewood. Ill: Dorsey Press, 1968.

Howard, I. P., \& Templeton, W. B. Human spatial orientation. London: Wiley, 1966.

Krewson, W. E. The action of the extraocular muscles. A method of vectoranalysis with computations. Transactions of the American Optical Society, 1950, 48, 443-486.

McLaughis, S. C. Parametric adjustment in saccadic eye movements. Perception \& Psychophysics, 1967, 2, 359-362.

PAAP, K. R. Perceptual consequences of post-tetanic-potentiation: An alternative explanation for adaptation to wedge prisms. Unpublished doctoral dissertation, University of Wisconsin, 1975.

PoLA, J. Voluntary saccades, eye position, and perceived visual direction. In R. H. Mantz \& J. W. Senders (Eds.), Eye movements and psychological processes. New Jersey: Lawrence Erlbaum, in press.

SCHÖNE, H. On the role of gravity in human spatial orientation. Aerospace Medicine, 1964, 35. 764-772.

SChUbert, G., \& Brecher, G. A. Über optische Lokalisation und Augenstellung bei vor-ruchwartsneigung oder exzentrisher Rotation des Korpers. Zeitschrift für Sinnesphysiologie, 1934, 65, 1-26.

WADE, N. J. Effects of prolonged tilt on visual orientation. Quarterly Journal of Experimental Psychology, 1970, 22, 423-439.

WALLACH, H. Informational discrepancy as a basis of perceptual adaptation. In S. J. Freedman (Ed.), The neuropsychology of spatially oriented behavior. Homewood, Ill: Dorsey Press, 1968.

(Received for publication March 29, 1976; revision accepted October $13,1976$. ) 